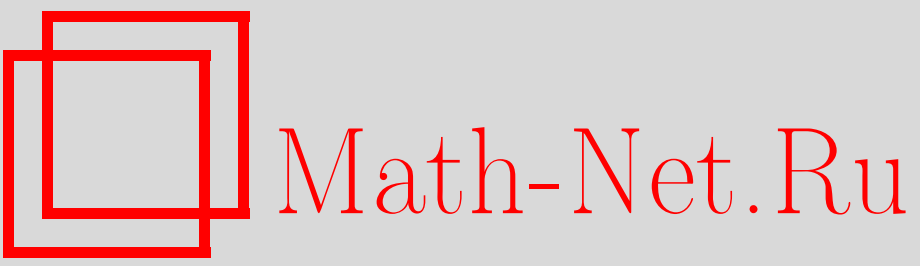

К. Клейн, Изомонодромийный подход к граничным задачам для уравнения Эрнста, ТМФ, 2003, том 134, номер 1, 85-100

DOI: https://doi.org/10.4213/tmf142

Использование Общероссийского математического портала Math-Net.Ru подразумевает, что вы прочитали и согласны с пользовательским соглашением

http://www . mathnet.ru/rus/agreement

Параметры загрузки:

IP: 35.173 .219 .149

26 апреля 2023 г., 08:00:05 
ТЕОРЕТИЧЕСКАЯ

И МАТЕМАТИЧЕСКАЯ

ФИЗИКА

Том 134, № 1

январь, 2003

(C) 2003 г.

К. Клейн*

\title{
ИЗОМОНОДРОМИЙНЫЙ ПОДХОД К ГРАНИЧНЫМ ЗАДАЧАМ ДЛЯ УРАВНЕНИЯ ЭРНСТА
}

\begin{abstract}
Свойства изомонодромии тета-функциональных решений уравнения Эрнста и асимптотическое разложение по спектральному параметру используются для установления между метрическими функциями и их производными алгебраических соотношений, которые налагаются соответствующей римановой поверхностью. Этими соотношениями определяется, какие классы граничных задач могут быть решены на данной поверхности. Подробно изучен случай римановых поверхностей низшего рода.
\end{abstract}

Ключевые слова: общая теория относительности, точные решения, изомонодромийные деформации.

\section{1. ВВЕДЕНИЕ}

Стационарные аксиально-симметричные уравнения Эйнштейна в вакууме играют важную роль в астрофизике, поскольку их решения могут описывать внешнее гравитационное поле звезд и галактик. Уравнения в соответствующих областях, заполненных материей, по-видимому, не являются интегрируемыми, однако Мэйсон [1] и Белинский и Захаров [2] доказали полную интегрируемость вакуумных уравнений в формулировке Эрнста [3], т.е. тот факт, что эти уравнения могут рассматриваться как условие интегрируемости переопределенной линейной системы дифференциальных уравнений для некоторой матрично-значной функции $\Phi$. Поскольку уравнение Эрнста является эллиптическим дифференциальным уравнением в частных производных, следует рассматривать граничные задачи, которые обычно задаются на границах областей, заполненных материей, и в идеале индуцируются решением уравнений Эйнштейна, включающих взаимодействие с материей. В случае инфинитезимально тонких дисков, которые рассматриваются как модели некоторых галактик или материи в дисках аккреции черных дыр, уравнения в заполненных материей областях редуцируются к обыкновенным дифференциальным уравнениям, решения которых дают граничные значения.

Стандартным подходом к решению граничных задач для интегрируемых уравнений являются методы Римана-Гильберта. Однако чтобы сформулировать задачу Римана-Гильберта для уравнения Эрнста, требуется поставить задачу Коши, которая явля-

\footnotetext{
*Max-Planck-Institut für Physik, München, Germany
} 
ется некорректной для эллиптических уравнений. В работе [4] было показано, что задачи Римана-Гильберта с рациональными скачками значений калибровочно-эквивалентны скалярной задаче на гиперэллиптической римановой поверхности. Эту задачу можно решить в рамках конечнозонных решений Короткина [5]. Таким образом представляется, что лучший способ решить граничные задачи - непосредственно использовать тета-функциональные решения. Первые пылевые диски были обнаружены в рамках такого подхода авторами работ [6] и [7]. Поскольку в пределах этого класса можно задать произвольную вешественнозначную функцию $G$ и набор параметров (точки ветвления гиперэллиптической поверхности), очевидно, что на поверхности конечного рода можно решить только некоторые классы граничных задач. При комплексном потенциале Эрнста в общем случае потребовались бы решения с двумя свободными функциями.

В статье [8] мы использовали алгебраический подход и соответствуюшую тета-функциональным решениям систему Пикара-Фукса для установления соотношений между потенциалом Эрнста и его производными, которые зависят только от характеристических величин римановой поверхности, но не от функции $G$. Недостатком этого подхода является то, что на поверхности рода $g$ он приводит к системе $g$ дифференциальных уравнений, и поэтому сложно решить, совместны ли определенные граничные значения с этими условиями.

В настоящей работе мы используем предложенный Короткиным и Николаи [9] подход к уравнению Эрнста на основе изомонодромии, адаптированный к алгебро-геометрическим решениям в работе [10], где использовался тот факт, что логарифмическая производная матрицы $\Phi$ по спектральному параметру $K$ является в некоторой калибровке рациональной функцией от $K$. Мы вычислим матричную функцию $\Phi_{K} \Phi^{-1}$ для поверхности произвольного рода и установим не зависяшие от функции $G$ соотношения между ее компонентами. Разлагая эти соотношения по спектральному параметру, можно определить матрицу $\Phi$ непосредственно из линейной системы для уравнений Эрнста и получить в результате иерархию тождеств между потенциалом Эрнста, метрическими функциями и их производными. Мы подробно обсудим случай низших родов.

Структура данной статьи такова. В разделе 2 приводятся важные факты, касающиеся уравнения Эрнста, его линейной системы и решений на гиперэллиптических римановых поверхностях. В разделе 3 определяются изомонодромийные свойства этих решений и устанавливаются соотношения, независимые от функции $G$. В разделе 4 мы разложим эти соотношения по спектральному параметру и с помощью линейной системы найдем связь между матрицей $\Phi$ и потенциалом Эрнста. Случаи родов 0,1 и 2 обсуждаются в разделе 5 . В разделе 6 суммируются основные результаты и делаются выводы.

\section{2. УРАВНЕНИЕ ЭРНСТА И ГИПЕРЭЛЛИПТИЧЕСКИЕ РИМАНОВЫ ПОВЕРХНОСТИ}

2.1. Уравнение Эрнста и линейная система. Метрику стационарного аксиально-симметричного вакуумного пространства-времени можно записать в форме Вей- 
ля-Льюиса-Папапетру (см. [11])) как

$$
d s^{2}=-e^{2 U}(d t+a d \phi)^{2}+e^{-2 U}\left(e^{2 k}\left(d \rho^{2}+d \zeta^{2}\right)+\rho^{2} d \phi^{2}\right),
$$

где $\rho$ и $\zeta$ - вейлевские цилиндрические координаты, а $\partial_{t}$ и $\partial_{\phi}-$ соответственно коммутируюшие асимптотически времениподобный и асимптотически пространственноподобный векторы Киллинга. Уравнения Эйнштейна в вакууме эквивалентны одному уравнению в частных производных для комплексного потенциала Эрнста $\mathcal{E}$ :

$$
\mathcal{E}_{z \bar{z}}+\frac{1}{2(z+\bar{z})}\left(\mathcal{E}_{\bar{z}}+\mathcal{E}_{z}\right)=\frac{2}{\mathcal{E}+\overline{\mathcal{E}}} \mathcal{E}_{z} \mathcal{E}_{\bar{z}}
$$

Здесь комплексная переменная $z$ обозначает $z=\rho+i \zeta$. Метрика (1) для заданного потенциала Эрнста получается из соотношений

$$
\begin{aligned}
e^{2 U} & =\operatorname{Re} \mathcal{E}, \\
a_{z} & =i \rho e^{-4 U}(\Im \mathcal{E})_{z}, \\
k_{z} & =2 \rho \frac{\mathcal{E}_{z} \overline{\mathcal{E}}_{z}}{(\mathcal{E}+\overline{\mathcal{E}})^{2}} .
\end{aligned}
$$

Типичные задачи с граничными условиями, представляющие интерес для уравнения Эрнста, являются смешанными нелинейными задачами. В случае инфинитезимально тонкого диска в экваториальной плоскости при обрашаюшемся в нуль давлении, так называемой пыли, их можно записать в виде

$$
\mathcal{E}_{\zeta}=-i \frac{R^{2}+\rho^{2}}{2 R \rho} \mathcal{E}_{\rho}+\frac{i}{R} e^{2 U}
$$

где $R=(a+1 / \Omega) e^{2 U}$ и $\Omega(\rho)-$ угловая скорость на диске, измеренная асимптотически невращающимся наблюдателем. Мнимая часть этого соотношения содержит производные метрических потенциалов только по $\rho$. Оно эквивалентно абелевому дифференциальному уравнению для $e^{2 U}$.

Уравнение Эрнста можно рассматривать как условие интегрируемости для переопределенной линейной дифференциальной системы для $(2 \times 2)$-матрично-значной функции $\Phi($ см. [5]):

$$
\begin{aligned}
& \Phi_{z}=\left\{\left(\begin{array}{cc}
N & 0 \\
0 & M
\end{array}\right)+\frac{K-i \bar{z}}{\mu}\left(\begin{array}{cc}
0 & N \\
M & 0
\end{array}\right)\right\} \Phi, \\
& \Phi_{\bar{z}}=\left\{\left(\begin{array}{cc}
\bar{M} & 0 \\
0 & \bar{N}
\end{array}\right)+\frac{K+i z}{\mu}\left(\begin{array}{cc}
0 & \bar{M} \\
\bar{N} & 0
\end{array}\right)\right\} \Phi,
\end{aligned}
$$

где $K$ - спектральный параметр на семействе римановых поверхностей $\mathcal{L}_{0}$, заданных уравнением

$$
\mu^{2}=(K-i \bar{z})(K+i z) .
$$


Специальное свойство уравнения Эрнста состоит в том, что спектральная кривая является “динамической”, т.е. ее точки ветвления зависят от физических координат $\rho$ и $\zeta$. Функции $M$ и $N$ зависят только от $z$ и $\bar{z}$, но не зависят от $K$ и имеют вид

$$
M=\frac{\mathcal{E}_{z}}{\mathcal{E}+\overline{\mathcal{E}}}, \quad N=\frac{\overline{\mathcal{E}}_{z}}{\mathcal{E}+\overline{\mathcal{E}}} .
$$

Из (8) следует, что определитель матрицы $\Phi$ имеет вид $\operatorname{det} \Phi=F(K) e^{2 U}$, где $F(K)-$ некоторая функция, не зависяшая от $z$ и $\bar{z}$.

Сушествование линейной системы (6) можно использовать для построения решений уравнения Эрнста с помошью следуюшей теоремы [5]. Обозначим точку на $\Sigma_{0}$ через $P=(K, \pm \mu(K))=K^{ \pm}$, а инволюцию, которая переставляет листы на $\mathcal{L}_{0}$, обозначим через $\sigma_{0}$.

ТЕОрема 1. Пусть матрииа $\Phi$ подчинена следующим условиям.

1. Она по крайней мере дважды дифференцируема по z и $\bar{z}$ и является голоморфной и обратимой в точках ветвления $\xi=-i z$ и $\bar{\xi}$, так что логарифмическая производная $\Phi_{z} \Phi^{-1}$ имеет полюс в точке $\xi$, а $\Phi_{\bar{z}} \Phi^{-1}-$ в точке $\bar{\xi}$.

2. Все сингулярности $\Phi$ на $\mathcal{L}_{0}$ таковы, что логарифмические производные $\Phi_{z} \Phi^{-1}$ и $\Phi_{\bar{z}} \Phi^{-1}$ являются голоморфными.

3. Матрица $\Phi$ подчинена условию редукиии

$$
\Phi\left(P^{\sigma_{0}}\right)=\sigma_{3} \Phi(P) \sigma_{1},
$$

где $\sigma_{1}, \sigma_{3}-$ матриць Паули.

4. Условие нормировки и вещественности имеет вид

$$
\Phi\left(P=\infty^{+}\right)=\left(\begin{array}{cc}
\overline{\mathcal{E}} & 1 \\
\mathcal{E} & -1
\end{array}\right) .
$$

Тогда функиия $\mathcal{E}$ является решением уравнения Эрнста.

ЗАМЕчАниЕ 1 . Если в уравнении $(9)$ вместо $\sigma_{1}$ использовать произвольную не зависящую от $z$ и $\bar{z}$ обратимую матрицу, теорема все равно будет выполняться, а потенциал Эрнста в (10) не изменится. Выбор $\sigma_{1}$ является выбором калибровки, но не фиксирует ее полностью. Остающаяся свобода определяется матрицей $C(K)=\kappa_{1} \hat{1}+\kappa_{2} \sigma_{i}$, $\kappa_{1}(\infty)=1, \kappa_{2}(\infty)=0$, действуюшей на $\Phi$ как $\Phi \rightarrow \Phi C(K)$.

2.2. Гиперэллиптические решения. Как было показано в работе [5], решения уравнения Эрнста можно построить на гиперэллиптической римановой поверхности $\mathcal{L}$ рода $g$, задаваемой уравнением

$$
\mu_{H}^{2}=(K-\xi)(K-\bar{\xi}) \prod_{i=1}^{g}\left(K-E_{i}\right)\left(K-\bar{E}_{i}\right),
$$

где точки ветвления $E_{i}=\alpha_{i}-i \beta_{i}$ не зависят от физических координат. Введем на $\mathcal{L}$ канонический базис циклов $\left(a_{i}, b_{i}\right)$ (мы используем систему разрезов из работы [8]), 
базис голоморфных дифференциалов $d \omega_{i}$ (дифференциалов первого рода), нормированных условием $\oint_{a_{i}} d \omega_{j}=2 \pi i \delta_{i j}$, отображение Абеля $\omega(P)=\int_{\xi}^{P} d \omega$ и матрищу $b$-периодов $\pi_{i j}=\oint_{b_{i}} d \omega_{j}$. Соответствуюшая кривой $\mathcal{L}$ тета-функция имеет вид [12]

$$
\Theta_{p q}(y \mid \Pi)=\sum_{m \in \mathbb{Z}^{g}} \exp \left\{\frac{1}{2}\langle\Pi(p+m),(p+m)\rangle+\langle p+m, y+2 i \pi q\rangle\right\} .
$$

Дифференциал третьего рода с полюсами в точках $P$ и $Q$, вычетами соответственно +1 и -1 и обрашающимися в нуль $a$-периодами будем обозначать $d \omega_{P Q}$.

Пусть $\Gamma$ - некоторый кусочно-гладкий контур на $\mathcal{L}$, обладающий тем свойством, что при $K \in \Gamma$ также $\bar{K} \in \Gamma$, и пусть $\ln G$ - некоторая непрерывная по Гёльдеру функция, подчиненная условию вешественности $G(\bar{K})=\bar{G}(K)$. Рассмотрим на $\mathcal{L}$ функции

$$
\psi\left(P_{H}\right)=\frac{\Theta\left(u+\omega\left(P_{H}\right)\right)}{\Theta\left(\omega\left(P_{H}\right)\right)} e^{I\left(P_{H}\right)}, \quad \chi\left(P_{H}\right)=\frac{\Theta\left(u+\omega\left(P_{H}\right)-\omega(\bar{\xi})\right)}{\Theta\left(\omega\left(P_{H}\right)\right)} e^{I\left(P_{H}\right)},
$$

где

$$
I\left(P_{H}\right)=\frac{1}{2 \pi i} \int_{\Gamma} \ln G d \omega_{P_{H} \infty^{-}},
$$

а вектор его $b$-периодов $u$ равен

$$
u=\frac{1}{2 \pi i} \int_{\Gamma} \ln G d \omega
$$

Функция $\chi$ не является однозначной на $\mathcal{L}$, поскольку она меняет знак на каждом разрезе $\mathcal{L}$. В работе [5] (см. также [13]) было показано, как удовлетворяющую условиям теоремы 1 матрицу $\Phi$ можно определить с помощью этих функций. Здесь мы фиксируем калибровку для матрищы $\Phi$ теми условиями, что ее единственные сингулярности даются контуром $Г$ и простыми полюсами в точках ветвления $E_{i}, \bar{E}_{i}$ и что определитель матрицы $\Phi$ постоянен по $K$. Это приводит к следуюшему предложению.

ПРЕДЛОЖЕНИЕ 1. Пусть $\Theta(u) \neq 0, \Theta\left(u+\omega\left(\infty^{-}\right)\right) \neq 0 u(2 \times 2)$-матрица $\Phi$ задана на $\mathcal{L}_{0}$ уравнением

$$
\begin{aligned}
\Phi(P)= & \sqrt{\frac{\operatorname{det}(\infty)}{\operatorname{det}(K)} \frac{e^{I\left(\infty^{+}\right) / 2}}{\Theta\left(u+\omega\left(\infty^{-}\right)\right) \Theta\left(u+\omega\left(\infty^{-}\right)+\omega(\bar{\xi})\right)} \times} \times \\
& \times\left(\begin{array}{c}
\Theta(u+\omega(P)) \Theta\left(u+\omega\left(\infty^{-}\right)+\omega(\bar{\xi})\right) e^{J / 2} \\
\Theta(u-\omega(P)) \Theta\left(u+\omega\left(\infty^{-}\right)+\omega(\bar{\xi})\right) e^{-J / 2} \\
\Theta(u+\omega(P)+\omega(\bar{\xi})) \Theta\left(u+\omega\left(\infty^{-}\right)\right) e^{J / 2} \\
-\Theta(u-\omega(P)+\omega(\bar{\xi})) \Theta\left(u+\omega\left(\infty^{-}\right)\right) e^{-J / 2}
\end{array}\right)
\end{aligned}
$$

при $P \sim \infty^{+}$, əдe

$$
\begin{gathered}
\operatorname{det}(K)=\Theta(u+\omega(P)) \Theta\left(u+\omega\left(P^{\sigma}\right)+\omega(\bar{\xi})\right)+\Theta\left(u+\omega\left(P^{\sigma}\right)\right) \Theta(u+\omega(P)+\omega(\bar{\xi})), \\
J=\frac{1}{2 \pi i} \int_{\Gamma} \ln G d \omega_{P P^{\sigma}}
\end{gathered}
$$

и $\sigma$ обозначает гиперэллиптическую инволючию. Тогда матрица $\Phi$ удовлетворяет условиям теоремы 1 и ее определитель постоянен по $\mathrm{K}$. 
ДокАЗАТЕЛЬСтво. Несложно проверить, что сингулярности матрищы $\Phi$, контур $\Gamma$ и точки ветвления поверхности $\mathcal{L}$ таковы, что условие 2 теоремы 1 выполнено. При $\xi \notin \Gamma$ и не совпадающем ни с одной из других точек ветвления $\mathcal{L}$ то же верно и для условия 1. Условие 3 и нормировка 4 выполняются по построению, тогда как вешественность $\mathcal{L}$ и условия вешественности на Г и $G$ обеспечивают выполнение условия вешественности 4 . С помошью трисекушего тождества Фея [12] можно эквивалентным образом представить $\operatorname{det}(K)($ см. [13]) как

$$
\operatorname{det}(K)=\frac{E\left(P, P^{\sigma}\right) E(\xi, \bar{\xi})}{E\left(P^{\sigma}, \xi\right) E(\bar{\xi}, P)} \Theta(u) \Theta(u+\omega(\bar{\xi}))
$$

откуда следует, что он может обрашаться в нуль, только если $P$ совпадает с точкой ветвления $E_{i}$ или $F_{i}$, т.е. $\Phi$ имеет сингулярности только вдоль Г. Это доказывает предложение 1.

В работе [8] мы использовали алгебраическую формулировку гиперэллиптических функций. Введем дивизор $X=\sum_{i=1}^{g} X_{i}$ как решение задачи обрашения Якоби $\omega(X)-$ $\omega(D)=u$ при $D=\sum_{i=1}^{g} E_{i}$ (мы предполагаем, что $X$ является неспециальным). Более того, мы используем ненормированые дифференциалы первого рода $d \nu_{i}(\tau)=\tau^{i-1} \times$ $d \tau / \mu_{H}(\tau), i=1, \ldots, g$, и дифференциалы третьего рода

$$
d \nu_{P Q}(\tau)=\left(\frac{\mu_{H}(\tau)+\mu_{H}(P)}{2 \mu_{H}(\tau)(\tau-P)}-\frac{\mu_{H}(\tau)+\mu_{H}(Q)}{2 \mu_{H}(\tau)(\tau-Q)}\right) d \tau
$$

Учитывая эти определения (здесь $P$ можно рассматривать как точку на гиперэллиптической поверхности), будем иметь

$$
\ln \left(\frac{\Theta(u+\omega(P))}{\Theta\left(u+\omega\left(P^{\sigma}\right)\right)} e^{J}\right)=-\sum_{i=1}^{g} \int_{F_{i}}^{\bar{X}_{i}} d \nu_{P P^{\sigma}}+\frac{1}{2 \pi i} \int_{\Gamma} \ln G d \nu_{P P^{\sigma}}
$$

где мы воспользовались тем, что $\omega(\bar{D})=K_{R}$ - риманов вектор. Другими словами, дивизор $\bar{X}$ является именно дивизором нулей тета-функции в $(17)$.

Поскольку $u$ вешественно, то $\omega(\bar{X})-\omega(D)=\omega(X)-\omega(\bar{D})$. Таким образом получаем, как и выше

$$
\ln \left(\frac{\Theta\left(u+\omega\left(P^{\sigma}\right)+\omega(\bar{\xi})\right)}{\Theta\left(u+\omega\left(P^{\sigma}\right)+\omega(\bar{\xi})\right)} e^{J}\right)=-\sum_{i=1}^{g} \int_{E_{i}}^{X_{i}} d \nu_{P P^{\sigma}}+\frac{1}{2 \pi i} \int_{\Gamma} \ln G d \nu_{P P^{\sigma}}
$$

По теореме Абеля из условия вещественности для $X$ следует существование мероморфной функции $R$ с полюсами в $\bar{X}+D$ и нулями в $X+\bar{D}$ (которая является рациональной в фундаментальном многоугольнике разрезанной поверхности):

$$
R(K)=\mathrm{const} \frac{\prod_{i=1}^{g}\left(K-E_{i}\right)\left(K-F_{i}\right)-Q(K) \mu(K)}{\prod_{i=1}^{g}\left(K-\bar{X}_{i}\right)\left(K-E_{i}\right)},
$$


где $Q(K)=x_{0}+x_{1} K+\cdots+x_{g-1} K^{g-1}-$ многочлен по $K$ с чисто мнимыми коэффициентами. Коэффициенты $x_{i}$ связаны с $X$ через алгебраическую (по $K$ ) систему

$$
\left(1-x^{2}\right) \prod_{i=1}^{g}\left(K-X_{i}\right)\left(K-\bar{X}_{i}\right)=\prod_{i=1}^{g}\left(K-E_{i}\right)\left(K-F_{i}\right)-Q^{2}(K)(K-\xi)(K-\bar{\xi}) .
$$

Их можно выразить в терминах $X$ с помощью соотношений $Q\left(X_{i}\right)\left(X_{i}-\xi\right)\left(X_{i}-\bar{\xi}\right)=$ $\mu_{H}\left(X_{i}\right), i=1, \ldots, g$, которые являются следствием уравнения (20). В дальнейшем мы будем полагать $x_{g-1}=x$. Чтобы решить систему для $X$ в зависимости от $x_{i}$, введем симметричные функции $S_{i}$ такие, что

$$
\prod_{i=1}^{g}\left(K-X_{i}\right)=S_{0}-K S_{1}+\cdots+(-1)^{g-1} K^{g-1}+K^{g} .
$$

Обозначим вешественную и мнимую части $S_{i}$ соответственно через $R_{i}$ и $I_{i}$. Можно использовать существование рациональной функции $R$, для того чтобы вычислить некоторые интегралы третьего рода как

$$
\frac{\Theta(u+\omega(P)) \Theta\left(u+\omega\left(P^{\sigma}\right)+\omega(\bar{\xi})\right)}{\Theta\left(u+\omega\left(P^{\sigma}\right)\right) \Theta(u+\omega(P)+\omega(\bar{\xi}))}=\exp \left(\int_{X+\bar{D}}^{\bar{X}+D} d \omega_{P^{\sigma} P}\right)=\frac{R(P)}{R\left(P^{\sigma}\right)} .
$$

При $P=\infty^{+}$из этого соотношения следует, что

$$
x=\frac{\mathcal{E}-\overline{\mathcal{E}}}{\mathcal{E}+\overline{\mathcal{E}}}
$$

Поскольку $u_{i}$ суть $b$-периоды интеграла $I$, они подчинены системе дифференциальных уравнений, так называемой системе Пикара-Фукса. Нетрудно проверить, что следующие уравнения, образующие подсистему уравнений Пикара-Фукса, являются прямым следствием определения $X$ :

$$
\begin{gathered}
\sum_{n=1}^{g} \frac{\left(X_{n}-\xi\right) X_{n}^{j}}{\mu_{H}\left(X_{n}\right)} X_{n, z}=0, \quad j=0, \ldots, g-2, \\
(\ln \mathcal{E})_{z}=\sum_{n=1}^{g} \frac{\left(X_{n}-\xi\right) X_{n}^{g-1}}{\mu_{H}\left(X_{n}\right)} X_{n, z} .
\end{gathered}
$$

Кроме того, нам потребуются так называемые корневые функции (см. [14]). Пусть $Q_{i}, i=1, \ldots, 2 g+2,-$ точки ветвления гиперэллиптической римановой поверхности $\mathcal{L}$ и $A_{j}=\omega\left(Q_{j}\right)$ при $\omega\left(Q_{1}\right)=0$. Кроме того, пусть $\left\{i_{1}, \ldots, i_{g}\right\}$ и $\left\{j_{1}, \ldots, j_{g}\right\}$ - два множества чисел из $\{1,2, \ldots, 2 g+2\}$. Тогда для произвольной точки $P \in \mathcal{L}$ выполнено равенство

$$
\frac{\Theta\left[K_{R}+\sum_{k=1}^{g} A_{i_{k}}\right](\omega(P))}{\Theta\left[K_{R}+\sum_{k=1}^{g} A_{j_{k}}\right](\omega(P))}=c_{1} \sqrt{\frac{\left(K-E_{i_{1}}\right) \ldots\left(K-E_{i_{g}}\right)}{\left(K-E_{j_{1}}\right) \ldots\left(K-E_{j_{g}}\right)}},
$$

где $c_{1}$ - константа, не зависяшая от $K$. 


\section{3. СООТНОШЕНИЯ ИЗОМОНОДРОМИИ}

В работе [10] приведен изомонодромийный подход к тета-функциональным решениям уравнения Эрнста в терминах спектрального параметра

$$
\gamma=\frac{2}{\xi-\bar{\xi}}\left(K-\frac{\xi+\bar{\xi}}{2}+\mu\right)
$$

Сектор изомонодромии определен соотношением

$$
\Phi_{\gamma} \Phi^{-1}=\sum_{i=1}^{2 N} \frac{A_{i}}{\gamma-\gamma_{i}}
$$

где $\gamma_{i}=\gamma\left(K_{i}\right)$, а $K_{i}$ и $A_{i}\left(\sum_{i=1}^{2 N} A_{i}=0\right)$ не зависят от $\xi, \bar{\xi}$. Сформулируем это соотношение непосредственно для спектрального параметра $K$ с помощью условия 3 теоремы 1 . Поскольку $\gamma\left(P^{\sigma}\right)=1 / \gamma(P)$, получим

$$
\Phi_{\gamma}(\gamma) \Phi^{-1}(\gamma)=-\frac{1}{\gamma^{2}} \sum_{i=1}^{2 N} \frac{\sigma_{3} A_{i} \sigma_{3}}{1 / \gamma-\gamma_{i}}
$$

Отсюда следует, что вместе с $\gamma_{i}$ также и $1 / \gamma_{i}$ должна быть полюсом $\Phi_{\gamma} \Phi^{-1}$. Рассматривая вычеты этой функции, можно переписать ее в виде

$$
\Phi_{\gamma}(\gamma) \Phi^{-1}(\gamma)=\sum_{i=1}^{N}\left(\frac{A_{i}}{\gamma-\gamma_{i}}+\frac{\sigma_{3} A_{i} \sigma_{3}}{\gamma-1 / \gamma_{i}}\right)
$$

С помошью (26) при $\mu_{i}=\mu\left(K_{i}\right)$ получаем

$$
\Phi_{K} \Phi^{-1}=\sum_{i=1}^{N}\left(\left(\begin{array}{cc}
A_{i 11} & 0 \\
0 & A_{i 22}
\end{array}\right) \frac{1}{K-K_{i}}+\left(\begin{array}{cc}
0 & A_{i 12} \\
A_{i 21} & 0
\end{array}\right) \frac{\mu_{i}}{\left(K-K_{i}\right) \mu}\right)
$$

где мы учли, что $\sum_{i=1}^{2 N} A_{i}=0$, откуда при приведенных вьше соотношениях следует, что $\sum_{i=1}^{N} A_{i}=0$ для диагональных элементов. Заметим, что матрицы $A_{i}$ бесследовые. Уравнение (30) дает обшую структуру $\Phi_{K} \Phi^{-1}$.

Решения, о которых говорилось в предыдушем разделе, были получены в работе [10] при рассмотрении случая, когда $N-g$ разрезов стягиваются, $E_{i} \rightarrow E_{j}$. Интеграл $\int_{\Gamma}$ возникает как непрерывный предел суммы $\sum_{i=g+1}^{N}$. Здесь мы хотим определить матрицы $A_{i}$ из $(30)$ в терминах точек ветвления $E_{i}$ и функции $G$ в этом предельном случае. Для этого мы работаем непосредственно с матрицей $\Phi$ из (14). Это приводит к следуюшей теореме. 
Tеорема 2. Пусть $\Xi:=\Phi_{K} \Phi^{-1}$ u $P \in \mathcal{L}$ nри $P \sim \infty^{+}$. Тогда $\Xi(P)$ имеет вид $\Xi_{11}(P)=-\Xi_{22}(P), \quad \Xi_{12}(P)=\bar{\Xi}_{21}(\bar{P}) u$

$$
\begin{aligned}
-4 \Xi_{11}= & \sum_{i=1}^{g}\left(\frac{1}{K-X_{i}}-\frac{1}{K-\bar{X}_{i}}\right)+\frac{Q(K) \mu_{H}(K)}{\prod_{i=1}^{g}\left(K-E_{i}\right)\left(K-F_{i}\right)} \times \\
& \times\left(-\sum_{i=1}^{g} \frac{d \omega_{X_{i} X_{i}^{\sigma}}}{d t}(P)-\sum_{i=1}^{g} \frac{d \omega_{\bar{X}_{i}} \bar{X}_{i}^{\sigma}}{d t}(P)-\frac{1}{\pi i} \int_{\Gamma}(\ln G)_{\tau} \frac{d \omega_{\tau \tau^{\sigma}}}{d t}(P) d \tau\right), \\
\Xi_{12}= & \frac{1-x}{2} \prod_{i=1}^{g} \frac{K-\bar{X}_{i}}{\sqrt{\left(K-E_{i}\right)\left(K-F_{i}\right)}} \times \\
& \times\left(-\sum_{i=1}^{g} \frac{d \omega_{\bar{X}_{i}} \bar{X}_{i}^{\sigma}}{d t}(P)-\frac{1}{2 \pi i} \int_{\Gamma}(\ln G)_{\tau} \frac{d \omega_{\tau \tau^{\sigma}}}{d t}(P) d \tau\right)
\end{aligned}
$$

Заметим, что $\Xi_{11}(K)=-\bar{\Xi}_{11}(\bar{K})$. Вычисления выполнены для $P \sim \infty^{+}$, поскольку знак функции $\chi$ не фиксирован единственным образом при любых значениях $P$. Это не влияет на диагональные компоненты матрицы $\Xi$, поскольку их можно записать как частные, в которых числитель и знаменатель линейны по $\chi$ (однако это не имеет места для недиагональных элементов).

ДОКАЗАТЕЛЬСТВо состоит из нескольких шагов.

1. Из (14) получаем

$$
\begin{aligned}
4 \Xi_{11}= & \partial_{K} \ln \frac{\Theta(u+\omega(P)) \Theta\left(u+\omega\left(P^{\sigma}\right)\right)}{\Theta(u+\omega(P)+\omega(\bar{\xi})) \Theta\left(u+\omega\left(P^{\sigma}\right)+\omega(\bar{\xi})\right)}+ \\
& +\frac{\Theta(u+\omega(P)) \Theta\left(u+\omega\left(P^{\sigma}\right)+\omega(\bar{\xi})\right)-\Theta\left(u+\omega\left(P^{\sigma}\right)\right) \Theta(u+\omega(P)+\omega(\bar{\xi}))}{\Theta(u+\omega(P)) \Theta\left(u+\omega\left(P^{\sigma}\right)+\omega(\bar{\xi})\right)+\Theta\left(u+\omega\left(P^{\sigma}\right)\right) \Theta(u+\omega(P)+\omega(\bar{\xi}))} \times \\
& \times \partial_{K} \ln \left(\frac{\Theta(u+\omega(P)) \Theta(u+\omega(P)+\omega(\bar{\xi}))}{\Theta\left(u+\omega\left(P^{\sigma}\right)\right) \Theta\left(u+\omega\left(P^{\sigma}\right)+\omega(\bar{\xi})\right)} e^{2 J}\right) \\
\Xi_{12}= & \frac{\Theta\left(u+\omega\left(\infty^{-}\right)+\omega(\bar{\xi})\right)}{\Theta\left(u+\omega\left(\infty^{-}\right)\right) \operatorname{det}(K)} \Theta(u+\omega(P)) \Theta\left(u+\omega\left(P^{\sigma}\right)\right) \partial_{K} \ln \left(\frac{\Theta(u+\omega(P))}{\Theta\left(u+\omega\left(P^{\sigma}\right)\right)} e^{J}\right)
\end{aligned}
$$

а также $\Xi_{11}(P)=-\Xi_{22}(P), \Xi_{12}(P)=\bar{\Xi}_{21}(\bar{P})$. Пользуясь хорошо известным фактом, что $\int_{A}^{B} d \omega_{C D}=\int_{C}^{D} d \omega_{A B}$, можно записать производную (17) по $K$ после интегрирования по частям (в предположении, что $\ln G$ обрашается в нуль в конечных точках $\Gamma$ ) как

$$
-\sum_{i=1}^{g} \frac{d \omega_{\bar{X}_{i}} \bar{X}_{i}^{\sigma}}{d t}(P)-\frac{1}{2 \pi i} \int_{\Gamma}(\ln G)_{\tau} \frac{d \omega_{\tau \tau^{\sigma}}}{d t}(P) d \tau
$$

Схожее соотношение можно получить для $K$-производной функции (18).

2. Для дальнейшего упрошения $\Xi_{11}$ покажем, что

$$
\partial_{K} \ln \frac{\Theta(u+\omega(P)) \Theta\left(u+\omega\left(P^{\sigma}\right)\right)}{\Theta(u+\omega(P)+\omega(\bar{\xi})) \Theta\left(u+\omega\left(P^{\sigma}\right)+\omega(\bar{\xi})\right)}=-\partial_{K}\left(\int_{\bar{X}}^{X}\left(d \omega_{P^{\sigma} Q}+d \omega_{P \widetilde{Q}}\right)\right)
$$


для $K$-независимых точек $Q$ и $\widetilde{Q}$. Таким образом, последнее выражение приобретает вид

$$
-\sum_{i=1}^{g}\left(\frac{1}{K-X_{i}}-\frac{1}{K-\bar{X}_{i}}\right),
$$

где мы использовали тот факт, что $d \omega_{P Q}+d \omega_{P \sigma} \widetilde{Q}=d \tau /(\tau-K)+\cdots$ (точками обозначены не зависяшие от $K$ члены). Из вида зависяших от $K$ членов следует, что их периоды обрашаются в нуль. Используя соотношения (19) и (21), получаем формулы (31) для элемента (33).

3. Аналогично рассматривается $\Xi_{12}$. Запишем множитель из (34) как

$$
\frac{\Theta\left(u+\omega\left(\infty^{-}\right)+\omega(\bar{\xi})\right) \Theta(u+\omega(P))}{\Theta\left(u+\omega\left(\infty^{-}\right)\right) \Theta(u+\omega(P)+\omega(\bar{\xi}))} \frac{1}{1+R(P)\left(R\left(P^{\sigma}\right)\right)^{-1}} .
$$

Отношение тета-функций равно взятому со знаком минус интегралу третьего рода

$$
\exp \left(\int_{\bar{X}}^{X} d \omega_{P^{\sigma} \infty^{+}}\right)=\exp \left(\int_{\bar{X}+D}^{X+\bar{D}} d \omega_{P^{\sigma} \infty^{+}}\right) \exp \left(\int_{\bar{D}}^{D} d \omega_{P^{\sigma} \infty^{+}}\right) .
$$

Второй интеграл можно вычислить, используя формулу (25) для корневых функций. Отсюда

$$
\exp \left(\int_{D}^{\bar{D}} d \omega_{P^{\sigma} \infty^{+}}\right)= \pm \prod_{i=1}^{g} \sqrt{\frac{K-F_{i}}{K-E_{i}}} .
$$

Неоднозначность в знаке отражает всего лишь неоднозначность знака функции $\chi$ при произвольном значении $P$. Для $P \sim \infty^{+}$знак фиксирован в соответствии с предельным случаем, когда рассматривается род 0 , где тета-функции не возникают. Учитывая уравнение (20), получаем для (38)

$$
\frac{1-x}{2} \prod_{i=1}^{g} \frac{K-\bar{X}_{i}}{\sqrt{\left(K-E_{i}\right)\left(K-F_{i}\right)}} .
$$

Таким образом мы получаем выражение (32), что и требовалось доказать.

ЗАмЕчАниЕ 2. Поскольку дифференциалы во второй строке (31) пропорциональны $\mu_{H}(K)$, написанное выше выражение отож дествляется с рациональной функцией (от $K$ ) на гиперэллиптической римановой поверхности, если рассматривать интеграл по $Г$ как предел суммы. Он может быть охарактеризован обычным способом при помощи своих полюсов. Несложно видеть, что вычеты в точках $K=X_{i}$ и $K=\bar{X}_{i}$ обрашаются в нуль: единственные члены, пропорциональные $1 /\left(K-X_{i}\right)$, за исключением таковых из первой строки в (31), возникают из-за

$$
\frac{d \omega_{X_{i} X_{i}^{\sigma}}}{d t}(P)=\frac{\mu_{H}\left(X_{i}\right)}{\left(K-X_{i}\right) \mu_{H}(K)}+\cdots,
$$

где точками обозначены члены, которые голоморфны при $X_{i} \sim K$; аналогичные соотношения вьполнены для $\bar{X}_{i}$. Таким образом с учетом $(32) \Xi_{12}$ очевидно регулярно в 
$\bar{X}_{i}$. Члены в $(31)$, пропорциональные $1 /\left(K-X_{i}\right)$, обрашаются в нуль при $K \rightarrow X_{i}$ ввиду (20). Аналогичный вывод можно сделать для членов, пропорциональных $1 /\left(K-\bar{X}_{i}\right)$. Следовательно, $\Xi$ можно записать в виде

$$
\begin{gathered}
4 \Xi_{11}=\sum_{i=i}^{g}\left(\frac{e_{i}}{K-E_{i}}+\frac{f_{i}}{K-F_{i}}\right)+\int_{\Gamma} \frac{A(\tau)}{K-\tau} d \tau \\
\Xi_{12}=\frac{1-x}{2 \sqrt{(K-\xi)(K-\bar{\xi})}}\left(\sum_{i=1}^{g}\left(\frac{c_{i}}{K-E_{i}}+\frac{d_{i}}{K-F_{i}}\right)+\int_{\Gamma} \frac{B(\tau)}{K-\tau} d \tau\right),
\end{gathered}
$$

как и следовало ожидать из обшего вида (30).

Форма уравнения (35) имеет тот недостаток, что периоды нормированных дифференциалов не вьпадают, как это происходит в (17). Интересно отметить, что тем не менее сушествуют два соотношения между компонентами $\Xi$, являющиеся алгебраическими по $X$, т.е. без контурных интегралов и периодов. Имеем

\section{СлЕДСтвиЕ 1. Выполняются соотношения}

$$
\begin{aligned}
-4 \Xi_{11}- & \sum_{i=1}^{g}\left(\frac{1}{K-X_{i}}-\frac{1}{K-\bar{X}_{i}}\right)=Q(K) \sqrt{(K-\xi)(K-\bar{\xi})} \times \\
\times & \left(\frac{2}{1-x} \frac{1}{\prod_{i=1}^{g}\left(K-\bar{X}_{i}\right)} \Xi_{12}+\frac{2}{1+x} \frac{1}{\prod_{i=1}^{g}\left(K-X_{i}\right)} \Xi_{21}\right), \\
(1+x) \Xi_{12} & \prod_{i=1}^{g}\left(K-X_{i}\right)-(1-x) \Xi_{21} \prod_{i=1}^{g}\left(K-\bar{X}_{i}\right)= \\
= & \frac{1}{2} Q(K) \sqrt{(K-\xi)(K-\bar{\xi})} \sum_{i=1}^{g}\left(\frac{1}{K-E_{i}}+\frac{1}{K-F_{i}}\right)- \\
& -\partial_{K}(Q(K) \sqrt{(K-\xi)(K-\bar{\xi})}) .
\end{aligned}
$$

ДокАЗАТЕЛЬСТВо. Соотношение (45) является прямым следствием теоремы 2. Для установления соотношения (46) используем $(34)$ и тот факт, что $\Xi_{12}(K)=\bar{\Xi}_{21}(\bar{K})$, откуда

$$
\begin{aligned}
& \frac{\Xi_{12}}{1-x} \prod_{i=1}^{g} \frac{\sqrt{\left(K-E_{i}\right)\left(K-F_{i}\right)}}{K-\bar{X}_{i}}-\frac{\Xi_{21}}{1+x} \prod_{i=1}^{g} \frac{\sqrt{\left(K-E_{i}\right)\left(K-F_{i}\right)}}{K-X_{i}}= \\
& \quad=\frac{1}{2} \partial_{K} \ln \frac{\Theta(u+\omega(P)) \Theta\left(u+\omega\left(P^{\sigma}\right)-\omega(\bar{\xi})\right)}{\Theta\left(u+\omega\left(P^{\sigma}\right)\right) \Theta(u+\omega(P)+\omega(\bar{\xi}))} .
\end{aligned}
$$

Члены в правой части (47) можно упростить, используя (21). С учетом (20) это приводит к (46). 


\section{4. АСИМПТОТИЧЕСКОЕ РАЗЛОЖЕНИЕ}

Мы хотим установить не зависяшие от функции $G$ соотношения между метрическими функциями и их производными на данной римановой поверхности. Идея состоит в том, чтобы установить желаемые соотношения, разлагая матрицу $\Phi$ из (6) по локальному параметру $\delta$ вблизи $\infty^{+}$. Для этого рассмотрим линейную систему (6) для матрицы $\widetilde{\Phi}$, заданной как $\widetilde{\Phi}(P):=\Phi\left(\infty^{+}\right)^{-1} \Phi(P)$ :

$$
\widetilde{\Phi}_{z}=\frac{1}{(\mathcal{E}+\overline{\mathcal{E}})^{2}}\left(\begin{array}{cc}
(\mathcal{E} \overline{\mathcal{E}})_{z} & (\mathcal{E}-\overline{\mathcal{E}})_{z} \\
\mathcal{E}^{2} \overline{\mathcal{E}}_{z}-\overline{\mathcal{E}}^{2} \mathcal{E}_{z} & -(\mathcal{E} \overline{\mathcal{E}})_{z}
\end{array}\right)\left(\sqrt{\frac{K-\bar{\xi}}{K-\xi}}-1\right) \widetilde{\Phi}
$$

которая имеет вид $\widetilde{\Phi}_{z}=\mathcal{K}(z, \bar{z})(\sqrt{(K-\bar{\xi}) /(K-\xi)}-1) \widetilde{\Phi}$, и аналогично для $\widetilde{\Phi}_{\bar{z}}$. Если разложить $\widetilde{\Phi}$ по $\delta: \widetilde{\Phi}=\hat{1}+\delta \Phi^{1}+\delta^{2} \Phi^{2}+\cdots$, получим

$$
\begin{aligned}
\Phi^{1} & =\int_{\kappa} d \kappa \mathcal{K} \frac{\xi-\bar{\xi}}{2}+C_{1}, \\
\Phi^{2} & =\int_{\kappa} d \kappa \mathcal{K} \Phi_{1}+\frac{1}{8} \int_{\kappa} d \kappa \mathcal{K}(3 \xi+\bar{\xi})(\xi-\bar{\xi})+C_{2}
\end{aligned}
$$

и т.д. с постоянными матрицами $C_{i}$. Интегралы $\int_{\kappa} d \kappa$ берутся по контуру в плоскости $z$ от некоторой точки $z_{0}$ до $z$. Это разложение можно использовать для определения разложения $\Xi$ по $\delta$. Для низшего порядка получаем следуюшую теорему.

ТеОРема 3. При g > 1 выполняются следующие соотношения:

$$
\begin{aligned}
& \Phi_{21}^{1}+\Phi_{12}^{1} \mathcal{E} \overline{\mathcal{E}}=\frac{\mathcal{E} \overline{\mathcal{E}}}{\mathcal{E}+\overline{\mathcal{E}}} \sum_{i=1}^{g}\left(X_{i}-\bar{X}_{i}\right), \\
& \Phi_{21}^{1}-\Phi_{12}^{1} \mathcal{E} \overline{\mathcal{E}}=-\frac{\mathcal{E}-\overline{\mathcal{E}}}{4}\left(\sum_{i=1}^{g}\left(E_{i}+\bar{E}_{i}\right)-\xi-\bar{\xi}\right)-\frac{\mathcal{E}+\overline{\mathcal{E}}}{2} x_{g-2} .
\end{aligned}
$$

Формулы верны также при $g=1$, если формально положить $x_{-1}=0$.

ДокАЗАТЕЛЬСтво. Записьвая $\Xi=\delta^{2} \Xi^{1}+\delta^{3} \Xi^{2}+\cdots$, получаем в первом порядке по $\delta$

$$
\Xi^{1}=-\Phi\left(\infty^{+}\right) \Phi^{1} \Phi^{-1}\left(\infty^{+}\right) .
$$

Калибровочное условие, согласно которому $\Phi$ имеет постоянный по $K$ определитель, в этом порядке выполнено, поскольку $\Phi^{1}$ бесследовая. Разлагая (45) и (46) по $\delta$, в низшем порядке получаем

$$
\begin{aligned}
-4 \Xi_{11}^{1}-\sum_{i=1}^{g}\left(X_{i}-\bar{X}_{i}\right) & =\frac{2 x}{1-x^{2}}\left((1+x) \Xi_{12}^{1}+(1-x) \Xi_{21}^{1}\right), \\
(1+x) \Xi_{12}^{1}-(1-x) \Xi_{21}^{1} & =\frac{x}{2}\left(\sum_{i=1}^{g}\left(E_{i}+\bar{E}_{i}\right)-\xi-\bar{\xi}\right)+x_{g-2} .
\end{aligned}
$$

Используя (53) и определение $x$ из (22), находим (51) и (52). 
ЗАмечАниЕ 3 . Компонента $\Phi_{12}^{1}$ с учетом (3) связана с метрической функцией $a$ как $\Phi_{12}^{1}=-i\left(a-a_{0}\right) / 2$. Константа $a_{0}$ определяется тем условием, что функция $a$ обрашается в нуль на регулярной части оси. Прямое следствие (51) и (52) состоит в том, что

$$
Z:=\left(a-a_{0}\right) e^{2 U}=\frac{i}{2} \sum_{i=1}^{g}\left(X_{i}-\bar{X}_{i}\right)+\frac{i x}{2\left(1-x^{2}\right)}\left(\sum_{i=1}^{g}\left(E_{i}+\bar{E}_{i}\right)-\xi-\bar{\xi}\right)+\frac{i x_{g-2}}{1-x^{2}}
$$

Эта формула впервые была приведена в [8]. Связь с функцией $\Phi_{21}^{1}$ является новой. Обе функции можно рассматривать как интеграл системы Пикара-Фукса (23) и (24), что можно видеть, дифференцируя (52) по $z$ (см., например, [8]).

\section{5. СЛУЧАИ НИЗШИХ РОДОВ}

Поучительно рассмотреть случаи низших родов в качестве иллюстрирации того, как можно использовать приведенные формулы для установления не зависящих от функции $G$ алгебраических соотношений между потенциалом Эрнста и некоторыми интегралами.

5.1. Статические решения. В случае рода 0 не возникает тета-функций, и потенциал Эрнста становится вешественным; решения являются статическими и принадлежат к так называемому классу Вейля. Полагая тета-функции в (14) равными 1 , легко проверить, что

$$
\Xi=\sigma_{1} \int_{\Gamma} \frac{A \mu(X)}{(X-K) \mu(K)} d X
$$

где мы отождествили $A$ с $(\ln G)_{\tau} /(4 \pi i)$. Кроме того,

$$
\Phi^{1}=\sigma_{3} \int_{z_{0}}^{z} d z^{\prime}(\xi-\bar{\xi}) U_{z}
$$

где постоянная матрица $C_{1}$ включена в $z_{0}$. В выбранной калибровке соотношение (30) приводит к

$$
\Phi\left(\infty^{+}\right) \Phi_{1} \Phi^{-1}\left(\infty^{+}\right)=\sigma_{1} \int_{\Gamma} A(X) \mu(X) d X
$$

т.е.

$$
\int_{z_{0}}^{z}(\xi-\bar{\xi}) U_{z} d z=\int_{\Gamma} A \mu(X) d X
$$

что выполняется тождественно. Значение $z_{0}$ можно фиксировать, например, сравнивая обе части уравнения на бесконечности. Поскольку род равен нулю, соотношения (51) и (52) выполняются тривиально.

4 Теоретическая и математическая физика, т. 134, № 1, 2003 г. 
5.2. Эллиптические решения. В случае рода 1 соответствуюшая риманова поверхность является эллиптической, что приводит к простейшим нестатическим решениям. Получаем

$$
\begin{aligned}
-4 \Xi_{11}= & \frac{1}{K-X}-\frac{1}{K-\bar{X}}-\frac{x}{(K-E)(K-F)}\left(\frac{\mu_{H}(X)}{K-X}+\frac{\mu_{H}(\bar{X})}{K-\bar{X}}-A_{X}-A_{\bar{X}}+\right. \\
& \left.+\frac{1}{i \pi} \int_{\Gamma}(\ln G)_{\tau}\left(\frac{\mu_{H}(\tau)}{K-\tau}-A_{\tau}\right) d \tau\right)
\end{aligned}
$$

где

$$
A_{y}=\oint_{a} \frac{\mu_{H}(y)}{\mu_{H}(t)(t-y)} d t / \oint_{a} \frac{d t}{\mu_{H}(t)} .
$$

Это означает, что с учетом (43)

$$
\begin{aligned}
& e=-\frac{1}{E-F}\left(X-\bar{X}-x A_{X}-x A_{\bar{X}}-\frac{x}{i \pi} \int_{\Gamma}(\ln G)_{\tau}\left(\frac{\mu_{H}(\tau)}{\tau-E}+A_{\tau}\right)\right), \\
& f=-\frac{1}{F-E}\left(X-\bar{X}-x A_{X}-x A_{\bar{X}}-\frac{x}{i \pi} \int_{\Gamma}(\ln G)_{\tau}\left(\frac{\mu_{H}(\tau)}{\tau-F}+A_{\tau}\right)\right), \\
& A=-\frac{x}{E-F} \frac{(\ln G)_{\tau}}{2 \pi i} \frac{\mu_{H}(\tau)}{K-\tau}\left(\frac{1}{\tau-E}-\frac{1}{\tau-F}\right) .
\end{aligned}
$$

Мы здесь использовали $x \mu_{H}(X)=(X-E)(X-F)$. Более того,

откуда с учетом (32)

$$
\begin{aligned}
\Xi_{12}= & \frac{1-x}{2 \sqrt{(K-\xi)(K-\bar{\xi})}} \frac{K-\bar{X}}{(K-E)(K-F)} \times \\
& \times\left(\frac{\mu_{H}(\bar{X})}{K-\bar{X}}-A_{\bar{X}}+\frac{1}{2 \pi i} \int_{\Gamma}(\ln G)_{\tau}\left(\frac{\mu_{H}(\tau)}{K-\tau}-A_{\tau}\right) d \tau\right),
\end{aligned}
$$

$$
\begin{aligned}
& c=\frac{1}{E-F}\left(\mu_{H}(\bar{X})-A_{\bar{X}}(E-\bar{X})+\frac{E-\bar{X}}{2 \pi i} \int_{\Gamma} d \tau(\ln G)_{\tau}\left(\frac{\mu_{H}(\tau)}{E-\tau}-A_{\tau}\right)\right), \\
& d=\frac{1}{F-E}\left(\mu_{H}(\bar{X})-A_{\bar{X}}(F-\bar{X})+\frac{F-\bar{X}}{2 \pi i} \int_{\Gamma} d \tau(\ln G)_{\tau}\left(\frac{\mu_{H}(\tau)}{F-\tau}-A_{\tau}\right)\right), \\
& B=\frac{(\ln G)_{\tau}}{2 \pi i} \frac{\mu_{H}(\tau)(\tau-\bar{X})}{(K-\tau)(\tau-E)(\tau-F)} .
\end{aligned}
$$

Для установления алгебраического соотношения между потенциалом Эрнста и метрическими функциями воспользуемся системой (20), которая в эллиптическом случае принимает вид

$$
\left(1-x^{2}\right) R_{0}=\alpha_{1}-\zeta x^{2}, \quad\left(1-x^{2}\right)\left(R_{0}^{2}+I_{0}^{2}\right)=\alpha_{1}^{2}+\beta_{1}^{2}-\left(\rho^{2}+\zeta^{2}\right) x^{2} .
$$

С учетом первого уравнения и равенства (56) во втором уравнении получим

$$
\left(1-x^{2}\right)\left(Z^{2}-\rho^{2}\right)-2 Z i x\left(\alpha_{1}-\zeta\right)=\beta_{1}^{2}-\rho^{2} .
$$

Это соотношение является полиномом по $Z$ и $x$. Оно выполнено при произвольном $G$ во всем пространстве-времени, а единственными свободными параметрами являются $\alpha_{1}$ и $\beta_{1}$. Схожее соотношение выполнено для $\Phi_{21}^{1}$ в силу (52), откуда следует, что в эллиптическом случае нужна только одна из величин $Z$ и $\Phi_{21}^{1}$. 
5.3. Случай рода 2. Для физических приложений специальный интерес представляют решения с отражательной симметрией в экваториальной плоскости $\zeta=0$. В работе [13] было показано, что такие решения можно найти на поверхностях четного рода; простейшие нестатические решения с экваториальной симметрией общего положения имеются на поверхности рода 2 при $E_{1}=-\bar{E}_{2}$. Именно в этом классе были найдены первые пылевидные диски [6], [7].

При $g=2$ система (20) принимает вид

$$
\begin{aligned}
\left(1-x^{2}\right) R_{1}= & \alpha_{1}+\alpha_{2}-\zeta x^{2}+x x_{0} \\
\left(1-x^{2}\right)\left(R_{1}^{2}+I_{1}^{2}+2 R_{0}\right)= & \left(\alpha_{1}+\alpha_{2}\right)^{2}+2 \alpha_{1} \alpha_{2}+\beta_{1}^{2}+\beta_{2}^{2}-x_{0}^{2}- \\
& -x^{2}\left(\rho^{2}+\zeta^{2}\right)+4 \zeta x x_{0} \\
\left(1-x^{2}\right)\left(R_{1} R_{0}+I_{1} I_{0}\right)= & \alpha_{1} \alpha_{2}\left(\alpha_{1}+\alpha_{2}\right)+\alpha_{1} \beta_{2}^{2}+\alpha_{2} \beta_{1}^{2}-\zeta x_{0}^{2}+\left(\rho^{2}+\zeta^{2}\right) x x_{0}, \\
\left(1-x^{2}\right)\left(R_{0}^{2}+I_{0}^{2}\right)= & \left(\alpha_{1}^{2}+\beta_{1}^{2}\right)\left(\alpha_{2}^{2}+\beta_{2}^{2}\right)-\left(\rho^{2}+\zeta^{2}\right) x_{0}^{2} .
\end{aligned}
$$

С учетом (52) получаем линейную зависимость $i x_{0}$ и $I_{1}$ от $\Phi_{21}^{1}$ и $Z$. Первые три уравнения из $(65)$ подобным же образом дают $R_{1}, R_{0}$ и $I_{0}$. Применяя их в последнем уравнении системы, получаем полиномиальное соотношение между $\Phi_{21}^{1}, Z$ и $x$, которое выполнено во всем пространстве-времени. В экваториально-симметричном случае в экваториальной плоскости это соотношение с учетом $E_{1}^{2}=: \alpha+i \beta$ и $Y:=-i(\mathcal{E}+\overline{\mathcal{E}}) \Phi_{21}^{1} /(\mathcal{E} \overline{\mathcal{E}})$ принимает вид

$$
\begin{aligned}
\left(\frac{1}{2} Y Z\right. & \left.-\frac{\alpha+\frac{\rho^{2}}{2}}{1-x^{2}}+\frac{\rho^{2}}{2}\right)^{2}(Y-Z)^{2}-\left(\frac{1}{2} Y Z-\frac{\alpha+\frac{\rho^{2}}{2}}{1-x^{2}}-\frac{\rho^{2}}{2}\right)^{2} x^{2}(Y+Z)^{2}= \\
= & \frac{\alpha^{2}+\beta^{2}}{1-x^{2}}(Y-Z)^{2}+\frac{\rho^{2}}{4}\left(1-x^{2}\right)\left(Y^{2}-Z^{2}\right)^{2} .
\end{aligned}
$$

Если выбрать контур интегрирования $\kappa$ в уравнении (49) вдоль оси из бесконечности к началу координат и далее в экваториальной плоскости к точке $(\rho, 0)$, то в результате интегрирования вдоль оси получается постоянная матрица $\Phi^{1}$, поскольку подынтегральное выражение в (49) обрашается в нуль. Интегрирование в диске дает интегралы по граничным соотношениям. Таким образом, соотношение (66) содержит только вклады граничных значений, две константы интегрирования и константы $\alpha$ и $\beta$. Остается проверить, можно ли выбрать эти константы таким образом, чтобы (66) имело место при некотором $\Omega(\rho)$ из (4).

\section{6. ЗАКЛЮЧЕНИЕ}

Используя алгебраический подход, мы определили функцию $\Xi=\Phi_{K} \Phi^{-1}$ для класса гиперэллиптических решений уравнения Эрнста для произвольного рода $\mathcal{L}$. Результаты применимы к отождествлению соотношений для $\Xi$, выполняюшихся на данной поверхности и не зависяших от функции $G$, параметризуюшей тета-функциональные решения. Используя асимптотическое разложение линейной системы для уравнения Эрнста по спектральному параметру, мы выразили матрицу $\Xi$ через интегралы от потенциала 
Эрнста. Найденные соотношения дают иерархию уравнений. Мы проиллюстрировали концепцию для случая низших родов: на поверхностях четного рода $g=2 n$ следует разлагать матрищу $\Phi$ до порядка $n$. Тогда соотношения (52) дают $2 n$ вешественных величин, которые можно использовать вместе с алгебраической системой $(20)$ для установления полиномиального соотношения между ними и $x=(\mathcal{E}-\overline{\mathcal{E}}) /(\mathcal{E}+\overline{\mathcal{E}})$. На поверхностях нечетного рода $g=2 n-1$ требуется только $2 n-1$ величин. Поскольку эти соотношения выполнены во всем пространстве-времени, они (как и их производные и определение дивизора $X$ ) должны также выполняться на некоторой границе, где решается граничная задача. Таким образом эти соотношения дают критерий разрешимости граничных задач на поверхностях конечного рода.

Найденные результаты представляют собой значительный прогресс по сравнению с результатами работы [13], где использовалась система тета-функциональных решений Пикара-Фукса, что на поверхности рода $g$ приводило к сложной системе $g$ дифференциальных уравнений. Такой подход не только значительно более сложен, чем использованный в данной статье, но и имеет дополнительный недостаток, состоящий в том, что для проверки разрешимости на данной поверхности граничные значения должны быть из класса $C^{g, \alpha}(\Gamma)$, тогда как в настояшем подходе они должны принадлежать только классу $C^{1, \alpha}(\Gamma)$. Остается проверить, можно ли в рамках очерченного подхода решить физически интересные граничные задачи, такие как задачи о дифференциально вращающихся пылевидных дисках или пылевидных дисках вокруг центральной черной дыры. Предметом будушего исследования является выяснение возможностей развития алгебраического подхода на случаи поверхностей высших родов.

Благодарности. Я благодарю Д. Короткина, который заинтересовал меня в данном предмете, за бесчисленные полезные замечания и подсказки. Работа была поддержана со стороны Schloessmann foundation.

\section{Список литературы}

[1] D. Maison. Phys. Rev. Lett. 1978. V. 41. P. 521.

[2] В. А. Белинский, В. Е. Захаров. ЖЭТФ. 1978. Т. 75. С. 1955.

[3] F. J. Ernst. Phys. Rev. 1968. V. 167. P. 1175; Phys. Rev. 1968. V. 168. P. 1415.

[4] C. Klein, O. Richter. Phys. Rev. D. 1998. V. 57. P. 857.

[5] Д. А. Короткин. ТМФ. 1988. Т. 77. С. 25.

[6] G. Neugebauer, R. Meinel. Phys. Rev. Lett. 1995. V. 75. P. 3046.

[7] C. Klein, O. Richter. Phys. Rev. Lett. 1999. V. 83. P. 2884.

[8] C. Klein. Phys. Rev. D. 2000. V. 63. P. 064033.

[9] D. Korotkin, H. Nicolai. Nucl. Phys. B. 1996. V. 475. P. 397.

[10] Д. А. Короткин, В. Б. Матвеев. Функц. анализ и его прилож. 2000. Т. 34. № 4. С. 18.

[11] D. Kramer, H. Stephani, E. Herlt, M. MacCallum. Exact Solutions of Einstein's Field Equations. Cambridge: Cambridge Univ. Press, 1980.

[12] J. D. Fay. Theta-functions on Riemann surfaces. Lect. Notes in Math. V. 352. Berlin: Springer, 1973.

[13] C. Klein, O. Richter. Phys. Rev. D. 1998. V. 58. P. 124018.

[14] E. D. Belokolos, A. I. Bobenko, V. Z. Enolskii, A. R. Its, V. B. Matveev. Algebro-Geometric Approach to Nonlinear Integrable Equations. Berlin: Springer, 1994. 